\title{
DOUBLE-MODE RR LYRAE VARIABLES: PULSATIONAL MASSES REVISITED
}

\author{
Giuseppe Bono \\ Osservatorio Astronomico di Trieste, Via G.B. Tiepolo 11, 34131 Trieste, Italy; \\ bono@oat.ts.astro.it \\ Filippina Caputo \\ Osservatorio Astronomico di Capodimonte, Via Moiariello 16, 80131 Napoli, Italy; \\ caputo@astrna.na.astro.it \\ Vittorio Castellani \\ Dipartimento di Fisica, Univ. di Pisa, Piazza Torricelli 2, 56100 Pisa, Italy; \\ vittorio@astr1pi.difi.unipi.it \\ and \\ Marcella Marconi \\ Dipartimento di Fisica, Univ. di Pisa, Piazza Torricelli 2, 56100 Pisa, Italy; \\ marcella@astr1pi.difi.unipi.it
}




\begin{abstract}
Double-mode RR Lyrae variables (i.e. radial variables which are simultaneously pulsating in both fundamental and first overtone modes) appear a fundamental tool for investigating the mass of old Population II Horizontal Branch (HB) stars. The most widespread method adopted for evaluating the masses of these objects is based on the Petersen (1973) approach, which relies only on pulsational periods $\left(P_{1} / P_{0}\right.$ vs. $\left.P_{0}\right)$ and therefore is independent of any preliminary evaluation of the reddening and/or of the distance modulus of the stellar cluster.

In this paper we supply an overview of the mass estimates and underline the role played by opacities as well as by full amplitude nonlinear models for removing the discrepancy between pulsational and evolutionary masses. On the basis of the comparison between the theoretical scenario and double-mode RR Lyrae stars belonging to selected Galactic globular clusters (IC4499, M3, M15, M68, NGC2419, NGC6426) we show that the $P_{1} / P_{0}$ vs. $P_{0}$ diagram can provide valuable constraints also on the luminosity of these variables.
\end{abstract}

Subject headings: globular clusters: individual (IC4499, M3, M15, M68, NGC2419, NGC6426) stars: horizontal branch - stars: oscillations - stars: variables: other 


\section{INTRODUCTION}

The evolution and the final fate of stellar structures is mainly governed by the amount of original mass. According to such a plain evidence, the large amount of present theoretical interpretations of the evolutionary status of stars and stellar systems is providing tight constrains on the mass of the investigated objects. An independent estimation of stellar masses would be of course of paramount interest since it would represent a prima facie evidence for the physical reliability of the adopted evolutionary scenario. As it is well known, radial pulsating structures offer such an opportunity for the simple reason that the pulsations are mainly governed by gravity. On this simple basis, the periods should depend, as they actually do, on pulsator masses and radii (i.e. on the stellar parameters M, L and Te). Jorgensen \& Petersen (1967) originally suggested that the occurrence of double-mode pulsators could give the unique opportunity to provide a straightforward evaluation of pulsator masses taking into account only the ratio between the fundamental $\left(P_{0}\right)$ and the first overtone periods $\left(P_{1}\right)$.

According to this scenario, Petersen (1973) introduced the diagram $P_{1} / P_{0}$ vs. $P_{0}$ (hereinafter referred to as $\mathrm{PD}$ ) as a suitable tool for estimating the actual mass value of double-mode pulsators. The application of this procedure to RR Lyrae stars dates back to Cox, King \& Hodson (1980). On the basis of the PD they found a mass value of about $M / M_{\odot}=0.65$ for the only double-mode RR Lyrae (RRd) known at that time (AQ Leonis, Jerzykiewicz \& Wenzel 1977). Since then the discovery of new RRd variables in several Galactic globular clusters, in the field (Clement et al. 1986, hereinafter referred to as C86) and in dwarf spheroidal galaxies (Nemec 1985a, Kaluzny et al. 1995) has brought on an interesting discussion for constraining their pulsational and evolutionary characteristics. Cox, Hodson \& Clancy (1983, hereinafter referred to as CHC) investigated the PD for RRd pulsators in the metal poor Oosterhoff II cluster M15 and derived a pulsational mass of the order of $\mathrm{M} / M_{\odot}=0.65$. The same authors suggested a mass of the order of $M / M_{\odot}=0.55$ for the two RRd pulsators belonging to M3, the prototype of intermediate metallicity Oosterhoff type I (Oo I) clusters. These results were subsequently confirmed by Nemec (1985b) and by C86 who found similar mass values for RRd variables in the Oo I cluster IC4499.

However, for the quoted HB pulsators current evolutionary theories foresee larger masses, namely $M / M_{\odot} \simeq 0.8$ and $\simeq 0.65$ for Oo II and Oo I clusters respectively (see Bono et al. 1996). Such a disturbing discrepancy between the masses of $\mathrm{RRd}$ variables determined from pulsational and from evolutionary theories was settled as soon as Cox (1991) found that pulsational models incorporating new and updated opacity evaluations were able to reconcile pulsational and evolutionary predictions. The settling of this long-standing discrepancy was thus regarded as an evidence for the reliability of the new opacity tables.

In this paper we present a new investigation of the Petersen approach which discloses some unexpected results and sheds new light on the matter. We show that opacity, as originally suggested by Cox $(1991,1995)$, is the key physical ingredient which produces the disagreement between pulsational and evolutionary masses. However, we also find that this discrepancy, even by 
using old opacities, can be consistently removed either by adopting a much finer spatial resolution in linear computations or by relying on detailed nonlinear models. Nevertheless, an exhaustive solution to the problem of RR Lyrae masses can only be achieved if both new opacities and full amplitude, detailed, nonlinear, nonlocal and time-dependent convective models are taken into account.

\section{MASSES AND LUMINOSITIES OF RRd VARIABLES}

During the last few years we have carried out an extensive survey of limiting amplitude, nonlinear models of RR Lyrae variables (Bono \& Stellingwerf 1994, hereinafter referred to as BS). The main purpose of this project is to examine the dependence of modal stability and pulsation behavior on astrophysical parameters (for complete details see Bono et al. 1996). As a by-product of this investigation we revisited the problem of pulsator masses by investigating the dependence of the Petersen diagram on the various assumptions governing theoretical calculations.

The sequences of static envelope models were analyzed in the linear nonadiabatic approximation (Castor 1971) and each model was required to cover the outer $90 \%$ of the stellar photospheric radii. The outer boundary condition was typically fixed at an optical depth of the order of 0.001. The linear models were constructed by neglecting convection and by adopting the analytical approximation of old Los Alamos "King" opacity tables provided by Stellingwerf (1975a,b). On the basis of these assumptions a typical coarse model is characterized by 100-150 zones and few percents of the total stellar mass. Complete details of the mass ratio between consecutive zones and the method adopted for constraining the hydrogen ionization region are given in Stellingwerf (1975a) and BS.

As a starting point, Fig. 1 shows the theoretical PD obtained for selected values of stellar masses and luminosities. The models plotted in this figure present a stable linear limit cycle in the first two modes. To understand the meaning of theoretical data displayed in this figure, we recall that linear models provide evaluations of periods independently of the actual limit cycle stability of a given mode. As a consequence, we have to bear in mind that a rather large amount of data in similar figures should be regarded as unphysical, since they supply the ratio $P_{1} / P_{0}$ even where either the fundamental or the first overtone modes present an unstable nonlinear limit cycle.

The period ratios of M15 RRd variables plotted in Fig. 1 were evaluated taking into account different estimates (Nemec 1985b; Kovacs, Shlosman \& Buchler 1986; Clement \& Walker 1990; Purdue et al. 1995). The error bar plotted in the lower right corner is referred to these measurements. The comparison between theoretical models and observational data, shown in the above figure, clearly supports previous results given in the literature under similar theoretical assumptions and discloses the occurrence of the "mass discrepancy problem". At the same time, Fig. 1 shows that at a given fundamental period the period ratio $P_{1} / P_{0}$ appears largely independent of the assumed luminosity level. 
In order to investigate the dependence of linear periods on the spatial resolution previously adopted, a new set of linear detailed models have been computed by adopting the prescriptions suggested by BS. The number of zones for these new sequences of models is increased by roughly a factor of two with respect to the coarse ones and ranges from 200 to 300. Fig. 2 shows the results of these new computations, disclosing that the "mass discrepancy problem" appears affected also by the method adopted to discretize the physical structure of the static envelope model. As a matter of fact, we find that periods provided by linear, nonadiabatic, radiative models constructed with a finer spatial resolution partially remove the degeneracy of the luminosity levels. Moreover, as a most relevant point, these calculations now suggest that the mass value of Oo II RRd variables should be of the order of $M / M_{\odot}=0.8$, whereas Oo I RRd variables should increase to about $M / M_{\odot}=0.70$, in much better agreement with evolutionary prescriptions (see Bono et al. 1996).

However, BS have already shown that linear periods are only a first, though good approximation of the pulsational periods obtained from a more appropriate nonlinear treatment of the pulsation. Thus the problem arises if linear predictions about RRd masses are preserved in the nonlinear approach. To properly address this fundamental theoretical question, Fig. 3 displays the results of several sequences of nonlinear, nonlocal and time-dependent convective models constructed by assuming the same equation of state and the same opacities adopted in the linear regime. According to the negligible influence of spatial resolution on nonlinear limiting amplitude characteristics and modal stability (BS and references therein) in order to speed up the calculations required by the nonlinear approach only coarse static envelope models were taken into account.

The dynamical behavior of the envelope models was examined for the first two modes and the static structures were forced out of equilibrium by perturbing the linear radial eigenfunctions with a constant velocity amplitude of $20 \mathrm{kms}^{-1}$. The method adopted for initiating nonlinear models unavoidably introduces a spurious component of both periodic and nonperiodic fluctuations which are superimposed to the pure radial motions. As a consequence, before the dynamical behavior approaches the limit cycle stability it is necessary to carry out extensive calculations. The fundamental and first overtone sequences have been integrated in time for at least 2,000 periods. The models located close to the fundamental blue edge and to the first overtone red edge were followed for a longer time interval (2,000-6,000 periods) since in these regions of the instability strip before the dynamical motions approach their asymptotic behavior a switch-over to a different mode could take place even after several thousand periods. The integration is generally stopped as soon as the nonlinear work term is vanishing and the pulsational amplitudes present a periodic similarity of the order of $10^{-(4 \div 5)}$.

Therefore it turns out that the decrease of theoretical points plotted in Fig. 3 is tightly connected with the morphology of the "OR region", since were taken into account only envelope models which present stable nonlinear limit cycles both in the fundamental and in the first overtone modes. Moreover, data in Fig. 3 reveal that the nonlinear PD differs intrinsically from the canonical linear PD. In fact in this new context the spurious theoretical points connected with models which present a unique stable limit cycle (fundamental or first overtone) have obviously disappeared. 
A direct interesting consequence of this new theoretical scenario is that the comparison between nonlinear periods and observational data can now give useful information on both stellar masses and luminosities of the pulsators. The reader interested to a thorough analysis concerning the evaluation of these parameters on the basis of $\mathrm{RRd}$ variables belonging to both Oo I and Oo II clusters is also referred to Cox (1995) and Walker (1995). In the evaluation of masses we eventually find that nonlinear results do not fully support linear indications. In fact, on the basis of nonlinear periods we obtain a stellar mass of $M / M_{\odot} \simeq 0.7$ for Oo II cluster pulsators, whereas for the RRd variables in IC4499 we estimate a mass of the order of $M / M_{\odot}=0.60$. As a consequence, the agreement found by relying on linear detailed models has to be regarded as an artifact of the computational procedure. Moreover, for M15 and M68 pulsators we find a luminosity around log $L / L_{\odot} \simeq 1.8$, which appears somewhat larger than the currently accepted evolutionary predictions.

Bearing in mind the present scenario, we now take into account the effects of the new opacities provided by Rogers \& Iglesias (1992) for temperatures higher than $10^{4}{ }^{\circ} \mathrm{K}$ and by Alexander \& Ferguson (1994) for lower temperatures. The reader interested in the method adopted for handling the new opacity tables is referred to Bono, Incerpi \& Marconi (1996). For the sake of conciseness, we briefly quote the mass evaluations obtained from linear computations: $M / M_{\odot}=$ 0.72, 0.60 (coarse models) and $M / M_{\odot}=0.78,0.65$ (detailed models) for pulsators in Oo II and Oo I clusters respectively. Fig. 4 shows nonlinear periods based on updated radiative opacities. The comparison with observational data is now pointing out a promising theoretical scenario since it predicts a stellar mass slightly greater than $M / M_{\odot}=0.8$ for RRd pulsators in Oo II clusters and a mass value around $M / M_{\odot}=0.65$ for RRd variables in IC4499. Both results are now in excellent agreement, within the error bar, with canonical evolutionary predictions. Data plotted in Fig. 4 also suggest a luminosity level of the order of $\log L / L_{\odot} \simeq 1.7$ for Oo II RRd variables, whereas the corresponding luminosity level for RRd variables in IC4499 falls between the computed luminosity levels at $\log L / L_{\odot}=1.61$ and 1.72 . The overall good agreement with evolutionary predictions, presented in Bono et al. (1996), shows that thanks to the updated physical input both pulsational and evolutionary theories converge to form a homogeneous scenario concerning the long debated question of RR Lyrae luminosity in globular clusters.

Finally, it is worth noting that the two RRd variables in M3 appear slightly more massive and more luminous than RRd variables in IC4499. According to current metallicity estimates for these clusters $\left([\mathrm{Fe} / \mathrm{H}]_{M 3}=-1.7,[\mathrm{Fe} / \mathrm{H}]_{I C 4499}=-1.5\right)$, even this finding appears again in satisfactory agreement with the evolutionary prescriptions.

\section{CONCLUSIONS}

In this paper we have revisited the approach based on the PD for determining the masses of RRd variables. It is shown that the pulsator masses evaluated through the comparison between periods obtained in a linear, nonadiabatic, radiative regime and observational data might be affected by substantial systematic errors. On the other hand, the periods provided by the surveys 
of nonlinear, nonlocal and time-dependent convective models point out that even though the discrepancy between linear and nonlinear periods has often been considered negligible, it plays a key role for properly defining the location of double-mode pulsators inside the Petersen diagram $\left(P_{1} / P_{0}\right.$ vs. $\left.P_{0}\right)$.

As a most relevant point, we found that a nonlinear Petersen diagram constructed taking simultaneously into account both nonlinear models and new radiative opacities provides valuable constraints not only on the stellar masses but also on the luminosities of $\mathrm{RRd}$ variables. The pulsational masses and luminosities of double-mode pulsators obtained in this new theoretical framework confirm the results recently provided by Cox (1995). The comparison with observational data of RRd variables in both Oo I and Oo II galactic globular clusters discloses a satisfactory agreement with current evolutionary and pulsational predictions. At the same time, this agreement supplies a new piece of evidence against the suggested anomaly of HB star luminosities. Further applications of this new approach for constraining the physical parameters of RRd variables belonging to the Galactic field, the central region of LMC and to dwarf spheroidal galaxies are under way.

It is a pleasure to thank A. Cox as referee for several valuable comments and for the pertinence of his suggestions on the original version of this paper. This work was partially supported by MURST, CNR-GNA and ASI. 


\section{REFERENCES}

Alexander, D. R. \& Ferguson, J. W. 1994, ApJ, 437, 879

Bono, G., Caputo, F., Castellani, V. \& Marconi, M. 1996, A\&AS, accepted

Bono, G., Incerpi, R. \& Marconi, M. 1996, ApJ Letters, accepted

Bono, G. \& Stellingwerf, R. F. 1994, ApJS, 93, 233 (BS)

Castor, J. I. 1971, ApJ, 166, 109

Clement, C. M. \& Nemec, J. M. 1990, JRASC, 84, 434

Clement, C. M., Nemec, J. M., Robert, N., Wells, T., Dickens, R. J. \& Bingham, E. A. 1986, AJ, $92,825(\mathrm{C} 86)$

Clement, C. M. \& Walker, I. 1990, AJ, 101, 1352

Cox, A. N. 1991, ApJ, 381, L71

Cox, A. N. 1995, in IAU JD 16, Astrophysical Applications of Powerful New Databases, eds. S.J. Adelman \& W.L. Wiese (San Francisco: ASP 78), 243

Cox, A. N., Hodson, S. W., \& Clancy, S. P. 1983, ApJ, 266, 94 (CHC)

Cox, A. N., King, S. P. \& Hodson, S. W. 1980, ApJ, 236, 219

Jerzykiewicz, M. \& Wenzel, W. 1977, Acta Astron. 27, 35

Jorgensen, H. E. \& Petersen, J. O. 1967, Zs. Astrophys., 67, 377

Kaluzny, J., Kubiak, M., Szymański, M., Udalski, A., Krzemiński, W. \& Mateo, M. 1995, A\&AS, 112,407

Kovacs, G., Shlosman, I. \& Buchler, J. R. 1986, ApJ, 307, 593

Nemec, J. M. 1985a, AJ, 90, 204

Nemec, J. M. 1985b, AJ, 90, 240

Petersen, J. O. 1973, A\&A, 27, 89

Purdue, P., Silberman, N. A., Gay, P. \& Smith, H. A. 1995, AJ, 110, 1712

Rogers, F. J. \& Iglesias, C. A. 1992, ApJS, 79, 507

Stellingwerf, R. F. 1975a, ApJ, 195, 441

Stellingwerf, R. F. 1975b, ApJ, 199, 705 
Walker, A. R. 1994, AJ, 108, 555

Walker, A. R. 1995, in IAU Colloq. 155, Astrophysical Applications of Stellar Pulsation, eds. R.S. Stobie \& P.A. Whitelock (San Francisco: ASP 83), 198

This preprint was prepared with the AAS LATEX macros v4.0. 


\section{4. $\quad$ Figure Captions}

Fig. 1. - Petersen diagram for linear, nonadiabatic and radiative periods. The RR Lyrae models were computed by adopting a fixed chemical composition $(Y=0.24, Z=0.0001)$ and the labeled values of stellar masses and luminosity levels. These envelope models were constructed by adopting the analytical opacity approximation provided by Stellingwerf (1975a,b). RRd variables observed in Oo I clusters are marked by open squares (IC4499, C86) and by stars (M3, CHC), whereas those observed in the Oo II clusters are displayed by open triangles (M68, Walker 1994), cross (NGC6426, Clement \& Nemec 1990) and diamond (NGC2419, Clement \& Nemec 1990). The period ratios of RRd variables belonging to M15 (open circles) were evaluated taking into account the estimates provided by Nemec (1985b), Kovacs, Shlosman \& Buchler (1986), Clement \& Walker (1990) and Purdue et al. (1995). The error bar plotted in the lower right corner is referred to these measurements.

Fig. 2. - Same as Fig. 1, but theoretical periods are referred to linear RR Lyrae models characterized by a finer spatial resolution.

Fig. 3. - Petersen diagram for limiting amplitude, nonlinear, nonlocal and time-dependent convective models. Only models which present a stable nonlinear limit cycle both in the fundamental and in the first overtone are displayed. These models were computed, for the labeled values of stellar masses and luminosity levels, by adopting the Stellingwerf analytical approximation and a coarse spatial resolution.

Fig. 4. - Same as Fig. 3, but theoretical periods are referred to nonlinear RR Lyrae models computed by adopting the new opacity tables provided by Rogers \& Iglesias (1992) for temperatures higher than $10^{4} \mathrm{~K}$, and by Alexander \& Ferguson (1994) for lower temperatures. 


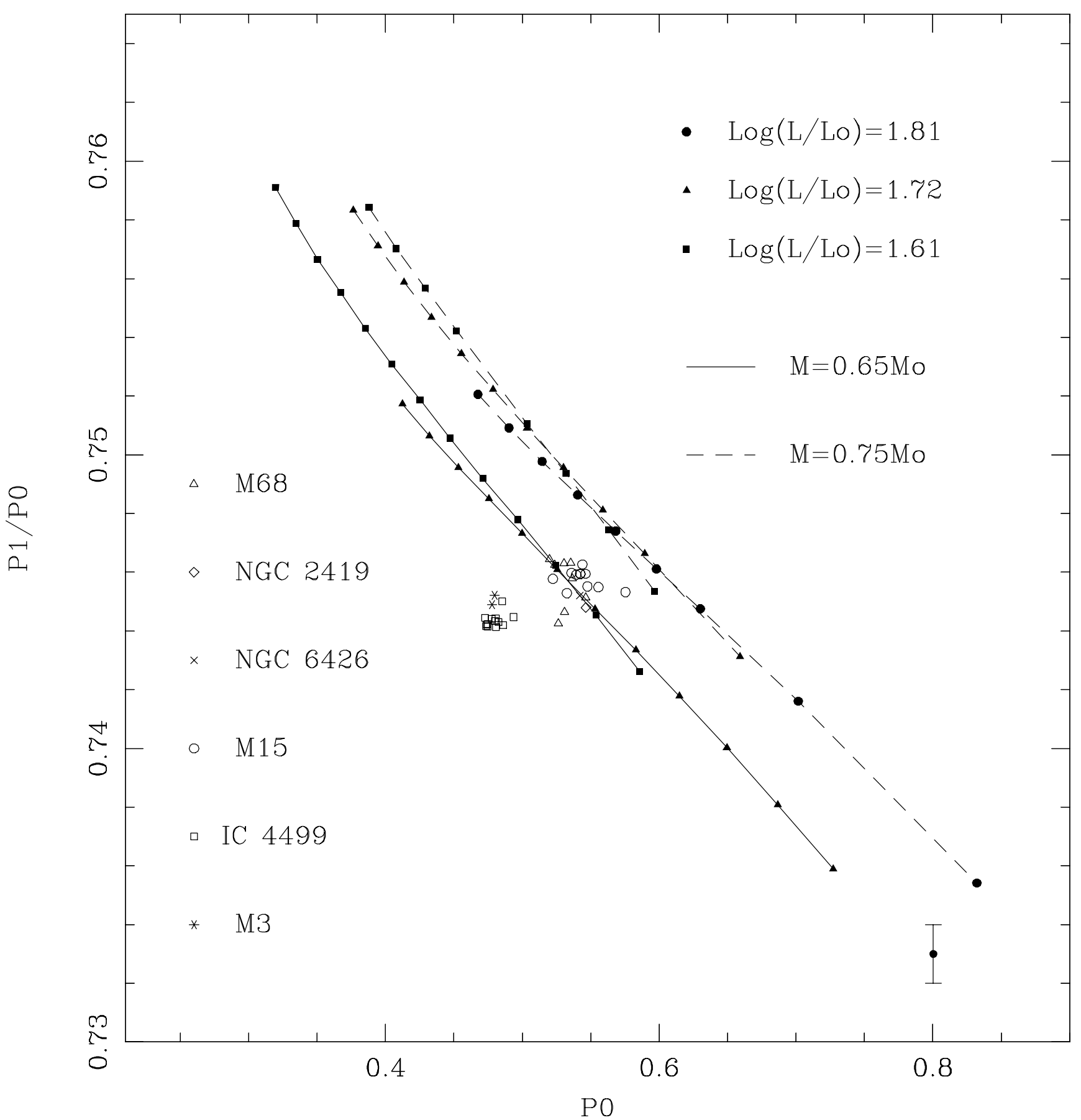




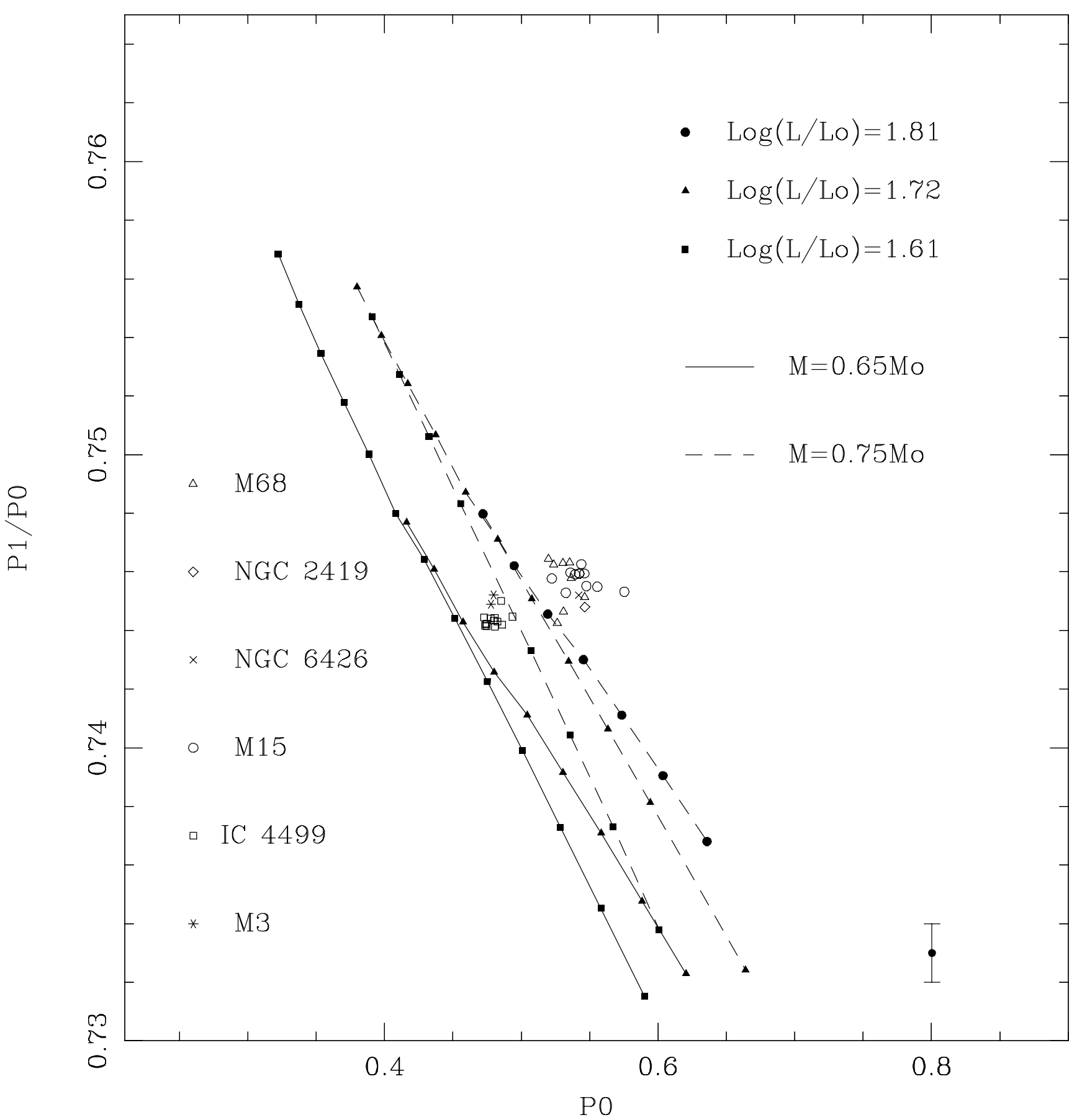




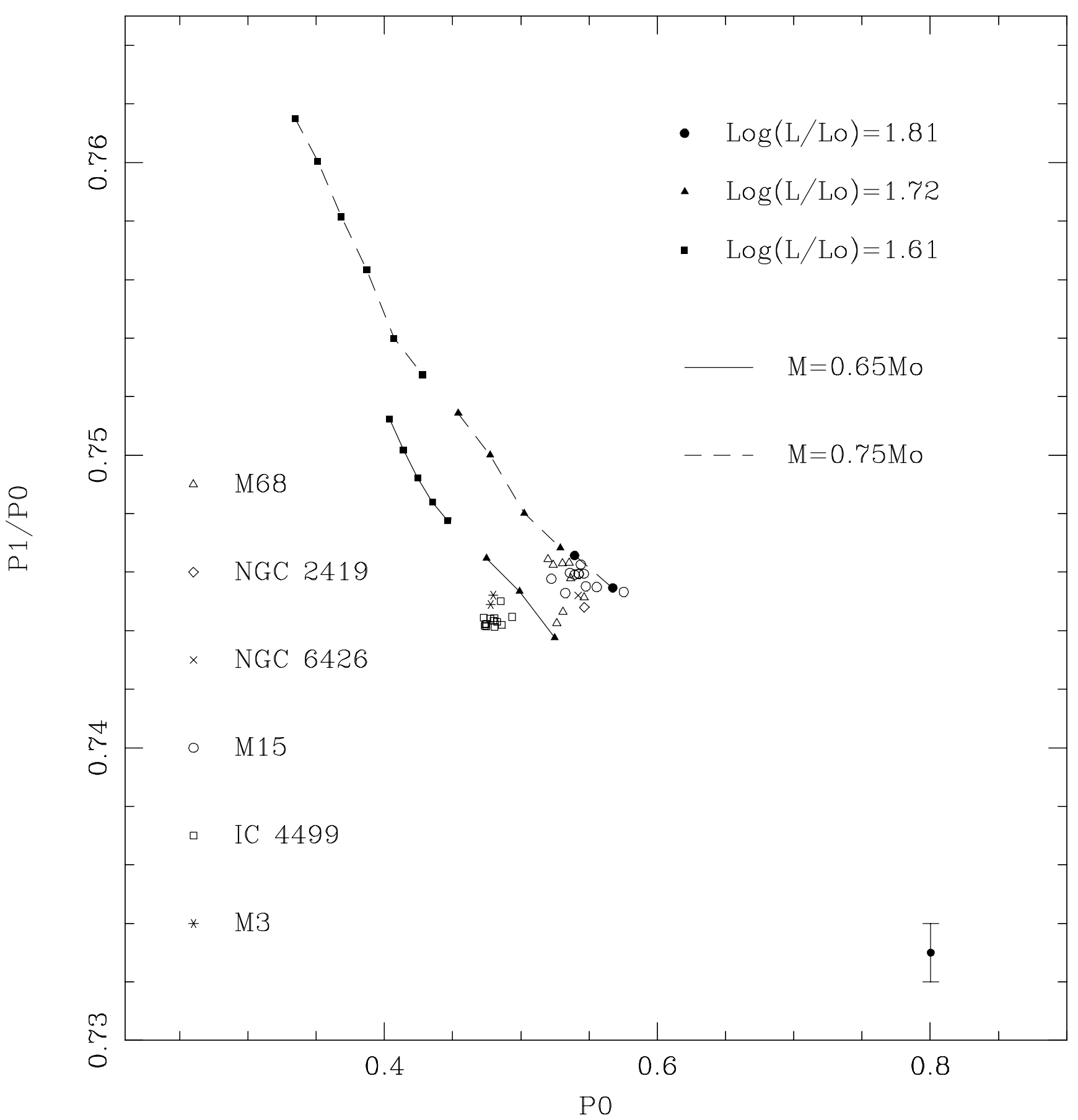




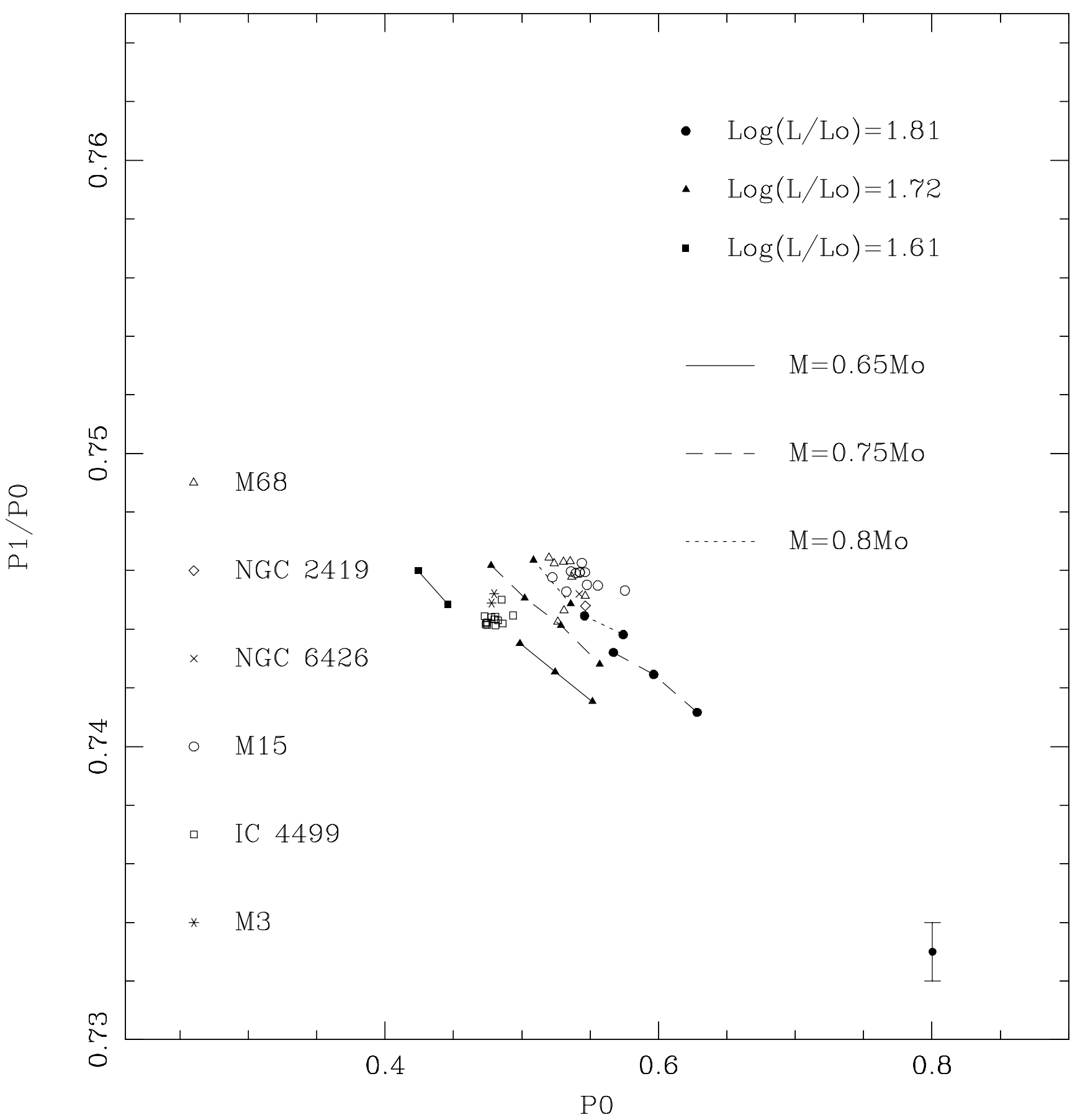

